\title{
MORTALIDADE DIFERENCIAL POR CAUSAS, SÃO PAULO, BRASIL, 1970: TABUAS DE VIDA DE MÚLTIPLO DECREMENTO*
}

Sabina Léa Davidson Gotlieb **

\begin{abstract}
GOTLIEB, S.L.D. Mortalidade diferencial por causas, Săo Paulo, Brasil, 1970: tábuas de vida de múltiplo decremento. Rev. Saúde públ., S. Paulo, 15:401-17, 1981.

RESUMO: Foram feitas consideraçóes sobre tábua de vida de múltiplo decremento, com o objetivo de avaliar a magnitude da atuação de alguns grupos de agravos à saúde nas probabilidades de morte, de sobrevivéncia e nas esperanças de vida dos residentes no município de São Paulo (Brasil), em 1970. A esperança de vida ao nascer foi igual a 60,12 anos no sexo masculino o 67,21 anos no sexo feminino. Os principais grupos de doenças em função dos ganhos que propiciariam à esperança de vida ao nascer, caso não tivessem sido fator de risco de morte, foram: no sexo masculino cardiovasculares; infecciosas e parasitárias; acidentes, envenenamentos e violências e os tumores malígnos; no sexo feminino - cardiovasculares; infecciosas e parasitárias; tumores malígnos e os acidentes, envenenamentos e violências. Levantou-se a hipótese de que o padrão de mortalidade no município de São Paulo, em 1970, refletiria a existência de problemas de saúde de uma populaçãc formada por setores distintos onde coexistiriam condições típicas adversas à saúde, ora de regiōes consideradas desenvolvidas, ora de regiöes em desenvolvimento.
\end{abstract}

UNITERMOS: Mortalidade, São Paulo, SP, Brasil.Tábuas de sobrevivência, múltiplo decremento. Esperança de vida:

\section{INTRODUÇAO}

O padrão de mortalidade segundo causas básicas de morte apresentado por uma regiāo pode refletir o grau de qualidade de vida ai existente e fornecer subsídios para uma política de saúde.

Considerando-se que a morte não é um evento repetitivo e nem atribuível a um único fator de risco ${ }^{4}$, devem ser levados em conta os vários riscos concomitantes e competitivos que atuam na vida de um ser humano. Neste sentido, a introdução da noção de riscos competitivos, que deram base à construção das tábuas de vida de múltiplo decremento, representam instru- mento analítico extremamente valioso em Epidemiologia e Saúde Pública. A tábua de vida de múltiplo decremento consegue descrever os efeitos separados e combinados das taxas de mortalidade, isto é, os decrementos da coluna dos vivos podem ser descritos em termos de algumas causas, em vez de se admitir uma única causa ${ }^{36}$.

Chiang ${ }^{4}$ admite que os vários riscos de morte atuam simultaneamente em cada indivíduo da população, havendo para cada risco uma correspondente força de mortalidade 8 . A soma destas é a força de mortalidade total, havendo uma razâo constante entre

* Resumo da tese "Mortalidade diferencial por causas, Săo Paulo, 1970: tábuas de vida de múltiplo decremento" apresentada à Faculda de de Saúde Pública da USP, em 1977, para a obtenção do título de Doutor.

* Do Departamento de Epidemiologia da Faculda de de Saúde Pública da USP - Av. Dr. Arnaldo. 715 - 01255 - São Paulo, SP - Brasil. 
GOTLIEB, S. L. D. Mortalidade diferencial por causas, São Paulo, Brasil, 1970: tábuas de vida de múltiplo decremento. Rev. Saúde públ., S. Paulo, 15:401-17, 1981.

força de mortalidade de uma causa e a total, em cada idade. Ao se eliminar uma causa de morte, a nova força de mortalidade estará relacionada à força de mortalidade total por meio da relação:

$u_{i \cdot j}(t)=u_{i}(t) \times \frac{D_{i}-D_{i \cdot j}}{D_{i}}$, onde

$u_{i \cdot j}(t)=$ força de mortalidade na idade $i$, excluída uma causa de óbito $\mathfrak{j}$, num instante $t$.

$u_{i}(t)=$ força de mortalidade total na idade $i$, num instante $t$.

$D_{i}=$ óbitos totais na idade $\mathrm{i}$.

$D_{i \cdot j}=$ óbitos totais na idade $i$, excluidos os óbitos pela causa $\mathrm{j}$.

Diante dessas informações e recomendações de vários autores $9,17,18,31,41$ sobre a validade do emprego das tábuas de vida de núltiplo decremento em análises de mortalidade, e dado o fato de a metodologia de Chiang * vir ao encontro da não existência da independência entre os riscos de morte na prática, surgiu o interesse em se conhecer a mortalidade diferencial por causas no municipio de São Paulo, em 1970, baseando-se em riscos competitivos.

O objetivo deste trabalho é avaliar a magnitude de atuação das doenças infecciosas e parasitárias, de tumores malígnos incluindo os neoplasmas do tecido linfático e órgãos hematopoéticos, das doenças cardiovasculares e dos acidentes, envenenamentos e violências nas probabilidades de morte, de sobrevivência e nas esperanças de vida, segundo sexo e idade, dos residentes no município de São Paulo, em 1970.

\section{MATERIAL E METODOS}

\subsection{Dados Necessários}

\subsubsection{População}

O contingente populacional residente no municipio de São Paulo em $1^{0}$ de julho de 1970 foi estimado por meio de interpolação das populações apresentadas nos Censos de $1960^{13}$ e $1970^{12}$. A taxa de crescimento médio mensal foi de $0,3652 \%$ ao mês, obtendo-se 0 total de $\mathbf{5 . 8 8 1 . 5 7 5}$ habitantes (10 de julho de 1970). Adotou-se a suposição de que a estrutura populacional por sexo e idade fosse a mesma do Censo de $1970 *$.

\subsection{2. obitos}

Os óbitos utilizados foram referentes apenas a 1970 e não ao número médio de óbitos do triênio (anos pré-censitário, censitário e pós-censitário) conforme recomendaçōes de autores ${ }^{10}$. Tal fato se deve à mudança na sistemática de classificação dos óbitos pelo Departamento de Estatística da Secretaria de Economia e Planejamento do Estado de São Paulo (atual Fundação SEADE), fonte oficial da informação dos óbitos do Estado de São Paulo. Esse órgão, até 1969 , tabulava os eventos vitais segundo local de ocorrência e, a partir de 1970, passou a classificá-los segundo o local de residência do falecido. Assim sendo, a eleição recaiu na utilização exclusiva dos óbitos de 1970, dada a inexistência de um critério único de classificação, no triênio.

Os grupos de causas básicas de morte analisados são os sugeridos pelas Nações Unidas para estudos de mortalidade ${ }^{3}$. Adotou-se este critério por estar baseado no comportamento das causas de morte em relação à ação sanitária, ou seja, sua maior ou menor resistência aos progressos médicos e programas de Saúde Pública. As causas estão apresentadas segundo a Lista $B$ da

- Fundação IBGE. Dados não publicados do Censo de 1970: Municípto de São Paulo. 
GOTLIEB, S. L. D. Mortalidade diferencial por causas, São Paulo, Brasil, 1970: tábuas de vida C.e múltiplo decremento. Rev. Saúde públ., S Paulo, 15:401-17, 1981.

Classificação Estatística Internacional de Doenças, Lesões e Causas de óbitos - $8^{a}$ Revisão $1965^{29}$.

\subsection{Metodologia}

A metodologia utilizada na construção da tábua de vida de múltiplo decremento diferencia-se daquela da tábua de vida, unicamente, na coluna - $\mathrm{QX}$ - proporção de mortes no intervalo. Neste caso, a probabilidade de morte é considerada líquida, isto é, admite-se que alguma causa específica de morte tenha sido eliminada da população; QX para então a ser estimada por

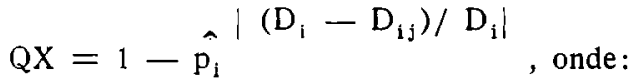

$\hat{p}_{i}=$ estimador de probabilidade de um indivíduo sobreviver de $x_{i}$ a $x_{i+n} e$ é igual a:

$\hat{p}_{i}=\frac{1-a^{\prime} N_{i} M_{i}}{1+\left(1-a^{\prime}{ }_{i}\right) N_{i} M_{1}}$ sendo que

$a_{i}^{\prime}=$ fator de separação ${ }^{*}$ para idade $i$

$\mathrm{N}_{\mathrm{i}}=$ intervalo de classe para o grupo etário considerado.

$M_{i}=$ coeficiente de mortalidade na idade $i$

$D_{i}=n^{\circ}$ de óbitos ocorridos na idade $\mathbf{i}$

$D_{i j}=n^{o}$ de óbitos ocorridos na idade i pela causa $\mathrm{j}$.

Neste trabalho foram adotados os seguintes simbolos:

$\mathrm{QX} .=$ probabilidade líquida de morte,

$P_{\text {ox. }}=$ probalidade hipotética de sobreviver, Ex. = esperança hipotética de vida, para as variáveis calculadas nas tábuas de vida de multiplo decremento, isto é, seriam hipotéticas pois pressupor-se-ia a inexistência de determinada doença como causa de morte.

\section{RESULTADOS E DISCUSSÃO}

\subsection{Esperança de vida}

Para ser avaliado o impacto de alguns agravos à saúde na esperança de vida dos residentes no municipio de São Paulo, em 1970 , construiu-se a tábua de vida, sem distinção de sexo. Com o resultado obtido 63,55 anos pode-se concluir que o município de São Paulo em 1970 apresentava-se em grau intermediário de desenvolvimento em Saúde Pública, encontrando-se no estágio IV, segundo Gabaldon ${ }^{14}$, com um atraso da ordem de 30 anos ao ser comparado com paises em alto grau de desenvolvimento em Saúde Pública. Segundo a classificação da OMS ${ }^{15}$, o municipio de São Paulo estava numa fase avançada de modernização e em transição para um padrão de baixa mortalidade, juntamente com $29 \%$ da população mundial de 1970.

Ramos ${ }^{33}$ comenta que o ganho na esperança de vida da populaçao do munıcípio de São Paulo entre 1899 e 1958 foi de 19 anos e tudo mostrava que este indicador poderia apresentar aumentos consideráveis nos anos seguintes a 1y58. Todavia tal previsão não foi confirmada pois o valor calculado para 1970 representou um acréscimo de 0,65 anos, em relação à esperança de vida ao nascer de 1958. É sabido que a esperança de vida ao nascer é altamente influenciada pelos valores assumidos pelo coeficiente de mortalidade infantil e analisando-se a tendência desse indicador na década de 1960 a 1970 verificou-se o grande aumento por ele apresentado $(62,94$ por mil nascidos vivos em 1960 passando a 89,46 por mil nascidos vivos, em $1970^{25}$ ), refletindo deterioração do nivel de saúde do município. Com este subsídio, pode-se entender a razão do mínimo aumento da esperança de vida ao nascer, na década analisada.

* Neste trabalho foram utilizados os valores do fator de separação calculadas por Oya 30. 
GOTLIEB, S. L. D. Mortalidade diferencial por causas, Såo Paulo, Brasil, 1970: tábuas de vida de múltiplo decremento. Rev. Saúde públ., S. Paulo, 15:401-17, 1981.

\subsection{Esperança de vida segundo sexo}

A esperança de vida, em 1970, nos sexos masculino e feminino, foi de 60,12 anos e 67,21 anos, respectivamente. $O$ ganho apresentado por este indicador entre 1950 e 1960 foi de 6,13 anos para homens e 8,47 anos para as mulheres ${ }^{35}$; entretanto, entre 1960 e 1970 houve decréscimo de 0,52 e 1,02 anos respectivamente, para homens e mulheres. Admite-se, então, que houve um processo de aprimoramento nas condiçōes de saúde do município de São Paulo entre 1950 e 1960 , mas tal processo não teve prosseguimento durante a década seguinte. Hipóteses podem ser levantadas, numa tentativa de explicar esta deterioração do nível de saúde do municipio de São Paulo. Uma, de cunho demográfico, é a existência de um grande contingente migratório, proveniente quer de zona rural quer de zona urbana mas de individuos com nivel educacional e econômico reduzido que se localizaram nas zonas periféricas do município, representando uma população com alto risco de morte 25 . Outro dado importante é o relativo ao salário minimo real que entre 1950 e 1960 teve aumento progressivo, mas no fim do decênio houve exacerbação da inflação e uma das medidas para contê-la foi a severa redução do salário mínimo real ${ }^{25}$. É indiscutível que com menor poder aquisitivo, as condições de saúde tendem a piorar, permitindo que as doenças encontrem um campo fértil para se expandir, contribuindo assim para uma maior mortalidade.

\subsection{Tábuas de vida de múltiplo decremento}

Assinalada a deterioração do nível de saúde do município de São Paulo, em 1970, as tábuas de vida de múltiplo decremento foram construídas para se aquilatar o importante papel que assumiram alguns agravos à saúde na mortalidade, analisando-se os mesmos segundo sexo e idade.

\subsubsection{Eliminando o Grupo das Doenças Infecciosas e Parasitárias}

Pelos dados oficiais, este grupo foi responsável por $11,6 \%$ dos óbitos totais, sendo que 0 risco de morrer de residentes no município de São Paulo, por este grupo de causas em 1970, foi de 10,3 por dez mil homens e 7,5 por dez mil mulheres (terceiro grupo de causa no sexo masculino e segundo no feminino, entre os mais importantes).

Comparando-se a probabilidade de morte em cada grupo etário ( $\hat{\mathrm{QX}}$ ) com a probabilidade liquida de morte ( $\hat{Q} X$.$) , isto é,$ excluindo este grupo de causas, sente-se o grande impacto destas causas no grupo de menores de um ano; a redução seria de $27,9 \%$ e $28,9 \%$ no sexo masculino e feminino, respectivamente.

A probabilidade de sobreviver $\left(\mathrm{P}_{\mathrm{ox}}\right)$ até um ano de idade, em média, aumentaria em $2,8 \%$, caso este grupo de causas não tivesse existido no sexo masculino.

A real esperança de vida (EX) e a hipotética (EX.) quando cotejadas mostram um ganho de vida em anos igual a 2,52 e 2,24 anos, respectivamente, para o sexo masculino e feminino, ganho de $4,2 \%$ a mais para os homens e $3,3 \%$ para as mulheres.

Apesar de ser hipotético este raciocinio, é importante lembrar que essas causas, na sua quase totalidade, são plenamente evitáveis. Informações de Leser e Barbosa ${ }^{25}$ demonstram que apenas $64 \%$ dos domicilios do município de São Paulo estavam ligados à rede pública de abastecimento de água e $40 \%$ ligados à rede pública de esgotos. Sabe-se ainda, que estes valores representam proporções médias e se pode afirmar que a menor cobertura de saneamento básico existe na zona periférica do município de São Paulo, onde há uma maior concentração de individuos em condições sócio-econômicas mais precárias. Esta combinação de fatores faz com que estas crianças das zonas periféricas estejam sujeitas a um maior risco de morte por doenças infec- 
GOTLIEB, S. L. D. Mortalidade diferencial por causas, São Paulo, Brasil, 1970: tábuas de vida de múltiplo decremento. Rev. Saúde públ., S. Paulo, 15:401-17, 1981.

ciosas. Laurenti 22, estudando crianças residentes no distrito de São Paulo, portanto excluídas as das zonas periféricas do município, observou que $51,8 \%$ das crianças falecidas em 1968/70 moravam em domicílios ligados à rede pública de abastecimento de água e apenas $30 \%$ das falecidas moravam em casas ligadas à rede de esgotos. Tal fato reforça a validade do binômio saneamento precário/alta mortalidade em crianças. Se medidas para solucionar este problema fossem energicamente acionadas, a idéia de mortes plenamente evitáveis deixaria de ser hipotética.

Levando-se em consideração que países desenvolvidos necessitaram, a partir de 1930, de dez anos para aumentar de 2,5 anos suas esperanças de vida ao nascer, configurando todos os esforços dirigidos ao aprimoramento do nivel de saúde de suas populaçōes, é importante frisar que o grande ganho de 2,5 anos, na esperança de vida dos residentes no município de São Paulo, poderia acontecer, proporcionalmente, em menor intervalo de tempo, caso as doenças infecciosas não fossem causa de morte. É importante lembrar entretanto que este ganho estaria ainda aquém do seu real valor, pois Laurenti 20 chama atenção para o fato de que a gastroenterite, como causa de óbito em menores de um ano, apareceu subenumerada em 39\%, nos dados oficiais de São Paulo em 1968/70. Portanto, esta eliminação deve ser considerada meta a ser atingida e o mais rapidamente possivel.

Se o saneamento é um problema, ião é o único a ser resolvido; há ainda a necessidade de ser comentada a existência do binômio "desnutrição e infecção". A Investigação Interamericana de Mortalidade na Infância 32 mostrou que em $28 \%$ dos óbitos de menores de um ano residentes no distrito de São Paulo existia a desnutrição como causa básica ou associada de morte e essa porcentagem se eleva a $48 \%$ no caso de óbitos entre um e 4 anos. $O$ fator desnutrição está intimamente ligado à redução do poder aquisitivo das classes sócio-econômicas menos favorecidas da população. $O$ salário mínimo real sofreu severa redução na década de 1960/70 e com isso as condições de alimentação da população foram prejudicadas quantitativa e, principalmente, qualitativamente 24. Estes fatos levam a pensar que além de solucionar problemas de saneamento, para dar mais anos de vida, há necessidade também de se melhorar a qualidade de vida, e um dos aspectos principais seria evitar a desnutrição, problema este realmente muito grave e de difícil solução pois há de existir mudanças consideráveis na estrutura social e econômica da população.

Com a finalidade de se obter uma estimativa mais fidedígna do risco de morrer por doenças infecciosas e parasitárias, dado o fato da real existência do mau preenchimento da parte médica do atestado de óbito ${ }^{32}$, as Nações Unidas ${ }^{3}$ preconizam que se agreguem os dados de óbitos por pneumonia ocorridos em menores de 5 anos, aos óbitos por doenças infecto-parasitárias. Foràn construídas, então, tábuas de vida com a exclusão dos óbitos por doenças infecciosas e pneumonias até 5 anos, e as probabilidades de morte estariam reduzidas em 50\% para menores de 1 ano $e$ nas idades de 1 a 3 anos, em $60 \%$. Como consequêencia, a esperança de vida estaria aumentada em 4,02 e 3,70 anos respectivamente para homens e mulheres. Estes ganhos representam anos de vida em pntencial sacrificados por causas plenamente evitáveis. Comparando estes ganhos de 4 anos com os dados de Gabaldon 14, chega-se à conclusão que bastaria que as doenças infecciosas e parasitárias não fossem causa de morte e o município de São Paulo estaria saindo do nível intermediário em desenvolvimento de saúde pública para o nivel elevado, representando uma região em que os seus habitantes estariam vivendo um padrão moderno de baixa mortalidade. 
GOTLIEB, S. L. D. Mortalidade diferencial por causas, São Paulo, Brasil, 1970; tábuas de vida de múltiplo decremento. Rev. Saúde públ., S Paulo, 15:401-17, 1981.

3.3.2. Eliminando o Grupo dos Tumores Malignos incluindo os Neoplasmas dos Tecidos Linfáticos e dos órgãos Hematopoéticos

Este grupo de causas foi responsável em 1970 , no município de São Paulo, por 9,74\% do total de ábitos masculinos e $11,32 \%$ dos femininos. O risco de morrer por câncer foi de 8,68 por dez mil homens e 7,30 por dez mil mulheres (quarto e terceiro grupo de causa de morte, respectivamente).

Ao se analisar a atuação do câncer nas probabilidades de morte, segundo sexo e idade, conclui-se que é mínima em menores de um ano, entretanto, com o decorrer das idades, as diferenças absolutas aumentam progressivamente, chegando ao máximo em 75 anos $(0,04386$ e 0,02930 , para os homens e mulheres respectivamente). $\mathrm{Na}$ ausência deste grupo de doenças, a probabilidade de morte estaria reduzida em torno de $10 \%$ na idade de 75 anos. $\mathrm{Na}$ análise, do ponto de vista relativo, faz-se notar a acentuada atuação dos neoplasmas em mulheres de 40 a 50 anos, pois haveria uma diferença de aproximadamente $25 \%$ entre a probabilidade real de morte e a hipotética. Nos homens, as maiores diferenças relativas são detectadas em idades um pouco mais avançadas ( 55 a 59 anos), com uma redução de $20 \%$ na probabilidade de morrer. Levin e col. ${ }^{27}$ comentam a mortalidade diferencial por sexo e idade, no sentido de que os neoplasmas aparecem mais precocemente nas mulheres, pois na idade de 20 a 40 anos a incidência feminina é três vezes maior do que a do sexo masculino; já no grupo etário 50 a 80 anos é mais comum entre os homens.

A probabilidade hipotética de sobrevivência, isto é, na ausência do câncer como causa de morte, mostra que dos cem mil nascidos vivos que iniciam a tábua de vida, chegariam aos 85 anos, $38 \%$ a mais de homens e $23 \%$ a mais de mulheres.

Se fosse possivel erradicar totalmente 0 câncer como causa de morte, as esperanças de vida ao nascer dos residentes no município de São Paulo, em 1970, seriam de 61,99 anos para os homens e 69,23 anos para as mulheres. As estimativas dos anos em potencial sacrificados mostram valores de 1,87 e 2,02 anos, respectivamente para os homens e mulheres. Os ganhos relativos são sempre maiores para os homens devido à sua menor esperança de vida. Ganhos diferentes, em função do sexo, também existem nos EUA to mas, relativamente, há uma ganho menor no municipio de São Paulo (Tabela 1). Uma das explicações possiveis seria a não existência, nos EUA, de um alto risco de morte por doenças infecciosas, em crianças, tal qual o que acontece entre nós; isto faz com que haja um maior contingente de indivíduos em idades mais avançadas, permitindo, talvez, uma exposição mais prolongada aos possiveis agentes cancerígenos, resultando dai maior incidência de casos de câncer e, consequientemente, maior mortalidade. Outros fatores explicativos seriam melhor diagnóstico da doença e maior precisão no preenchimento do atestado de óbito.

Sabe-se que a suposição da eliminação total dos tumores malígnos como fator de risco de morte, com os conhecimentos atuais, é impraticável e irreal mas deve existir para o sanitarista o objetivo de prevenir ou mesmo retardar a mortalidade por câncer. $O$ que poderia ser feito para, talvez, alcançar este objetivo é incentivar programas de "screening" e diagnóstico precoce dos casos e envidar esforços para que haja tratamento mais eficiente e eficaz dos casos diagnosticados. Com isso, diminuir-se-ia o coeficiente de mortalidade especifico por idade, especialmente nos mais jovens e aumentar-se-ia a sobrevivência dos pacientes com câncer que obtivessem tratamento específico. Esta atitude pode ser considerada prevenção secundária das doenças crônicas, isto é, detecção das doenças durante seu estágio inicial, ocasião em que um tratamento adequado poderia fazer cessar o seu progresso, evitando incapacidade física e morte prematura ${ }^{2}$ 
GOTLIEB, S. L. D. Mortalidade diferencial por causas, São Paulo, Brasil, 1970: tâbuas de vida de múltiplo decremento. Rev. Saúde públ., S. Paulo, 15:401-17, 1981.

T A B E L A 1

\begin{tabular}{|c|c|c|c|c|}
\hline Sexo & \multicolumn{2}{|c|}{ Masculino } & \multicolumn{2}{|c|}{ Feminino } \\
\hline Idade & $\begin{array}{c}\text { São Paulo } \\
1970\end{array}$ & $\begin{array}{c}\text { EUA * } \\
1.973\end{array}$ & $\begin{array}{c}\text { São Paulo } \\
1970\end{array}$ & $\begin{array}{c}\text { EUA * } \\
1973\end{array}$ \\
\hline 45 & 2.00 & 2,57 & 1,83 & 2,55 \\
\hline 50 & 1,92 & 2,47 & 1,76 & 2,35 \\
\hline 55 & 1,78 & 2,32 & 1,55 & 2,10 \\
\hline 60 & 1,56 & 2,10 & 1,34 & 1,80 \\
\hline $6 b$ & 1,37 & 1,81 & 1.10 & 1.48 \\
\hline
\end{tabular}

* Fonte: Statistical Bulletin 40.

Por outro lado, esforços devem ser difundidos no sentido de se incentivar programas de prevenção a nível primário, se factível, através de métodos simples de Saúde Pública. Segundo Doll 7 , mesmo não se conhecendo, ainda, o mecanismo pelo q̨ual o câncer é produzido na célula, sua prevenção torna-se uma possibilidade graças a estudos epidemiológicos que permitiram definir condições ambientais que agem como fatores de risco. Levin ${ }^{26}$ afirma que $40 \%$ a $50 \%$ dos tumores malignos serão preveníveis, em futuro próximo, caso se consiga aumentar acentuadamente 0 controle do hábito de fumar.

Já há evidências que $80 \%$ dos tumores, direta ou indiretamente, dependem de fatores ambientais, cabendo à Epidemiologia instrumento essencial da Saúde Pública investigar o papel dos perigos dos ambientes para 0 homem ${ }^{16}$ e identificar grupos de pessoas sujeitas a alto risco de câncer ${ }^{11,34}$.

Caso estas recomendações relativas à prevenção, diagnóstico e tratamento precoces viessem a ser executadas no município de São Paulo, respeitando as prioridades necessárias, ter-se-ia a possibilidade de reduzir a mortalidade por câncer, e o ganho hipotético na esperança de vida, aqui estimado, tornar-se-ia, em parte, realidade.

\subsubsection{Eliminando o Grupo das Doenças Cardiovasculares}

Compreendendo as doenças reumáticas do coração, as hipertensivas, as isquêmicas do coração e as cerebrovasculares, este grupo foi responsável por $28,17 \%$ dos óbitos masculinos e $32,68 \%$ dos femininos. O risco de morrer dos residentes no município de São Paulo, em 1970, foi de 25,13 por dez mil homens e 21,07 por dez mil mulheres, tornando-se o principal grupo de causas de morte, em ambos os sexos.

Caso estas doenças não fossem causa de morte, haveria uma grande redução das probabilidades de morrer. As diferenças absolutas entre a probabilidade real de morte e a hipotética são crescentes com $o$ aumentar das idades, em ambos os sexos, com maiores diferenças para os homens. As diferenças relativas, entretanto, são constantemente maiores para as mulheres, 
GOTLIEB, S. L. D. Mortalidade diferencial por causas, São Paulo, Brasil, 1970: tábuas de vida de múltiplo decremento. Rev. Saúde públ., S. Paulo, 15:401-17, 1981.

dado o menor risco de morrer apresentado por elas. Para os homens, a partir de 35 anos existiria uma redução minima de $20 \%$, chegando ao máximo de $50 \%$ na faixa etária de 75 a 79 anos. Nas mulheres, as reduções, nestas mesmas idades, seriam de $24 \%$ e $58 \%$, respectivamente.

As tábuas de vida de múltiplo decremento, com a exclusão das doenças cardiovasculares como causa de morte, mostram esperanças de vida ao nascer de 69,26 e 78,65 anos, respectivamente, para homens e mulheres. A comparação entre as esperanças de vida real e hipotética mostra ganhos acentuados de 9,14 e 11,44 anos; o número de anos ganhos é sempre maior para o sexo feminino, com valor máximo aos 40 anos (12,56 anos). Para os homens, o maior ganho se dá na idade de 45 anos (10,44 anos).

Visto que as doenças isquêmicas do coração e as cerebrovasculares constituem as duas mais importantes causas de óbitos, dentre as cardiovasculares, analisou-se, separadamente, a atuação de cada uma delas.

0 grupo de isquêmicas do coração representaram $12,69 \%$ dos óbitos masculinos e $11,62 \%$ dos femininos, com risco de morte de 11,31 por dez mil homens e 7,49 por dez mil mulheres.

Relativamente às probabilidades liquidas de morte, verifica-se a grande importância que assumem as doenças isquêmicas do coração na mortalidade masculina. $\mathrm{Na}$ sua ausência haveria uma redução de $11,44 \%$ na probabilidade de morrer de homens de 40 anos, sendo que a diferença absoluta é três vezes maior do que a redução absoluta das mulheres. Esta razão se mantém até $\mathbf{5 0}$ anos, e a partir dos 55 anos o valor da redução é duas vezes maior para homens.

A probabilidade de sobreviver do nascimento até aos 85 anos estaria aumentada em $67,43 \%$ e $39,05 \%$ respectivamente para os homens e mulheres, caso a doença isquêmica do coração não se consti- tuisse em causa de morte dos residentes no município, em 1970. Dos individuos que completaram 15 anos, $67,46 \%$ dos homens chegariam aos 65 anos e 80,10\% das mutheres, em lugar de $61,87 \%$ e $\mathbf{7 7 , 2 9 \%}$, respectivamente.

A esperança de vida ao nascer, com eliminação desse grupo de causas, seria de 62,90 anos para os homens e 69,71 anos para as mulheres.

O ganho em anos seria maior para os homens, com valor máximo de 3,23 anos, na idade de 40 anos.

As doenças cerebrovasculares, de comportamento epidemiológico segundo sexo e idade distinto quando comparado ao das doenças isquêmicas do coração, representaram 8,31\% dos óbitos masculinos e $11,27 \%$ dos femininos. Apesar de ser mais importante no sexo feminino, do ponto de vista relativo, quanto ao risco de morrer são os homens os mais suscetiveis $(7,41$ por dez mil homens e $\mathbf{7 , 2 7}$ por dez mil mulheres).

As probabilidade líquidas de morte apresentam reduções maiores para os homens exceto no último grupo etário quando há maior redução para mulheres. Relativamente, este grupo de causas é mais importante para as mulheres, dado o maior decréscimo percentual.

A probabilidade de um individuo de 15 anos chegar aos 65 anos, caso as doenças cerebrovasculares não fossem causa de óbito, seria de $65 \%$ (em vez de $61,87 \%$ ) para os homens e $80,03 \%$ (em vez de $77,29 \%$ ) para as mulheres.

As esperanças de vida, com eliminação desse grupo de causas de morte, seriam de 61,82 e 69,50 anos, respectivamente, para os homens e mulheres. Ganhos maiores existiriam na esperança de vida feminina, em todas as idades, sendo mais acentuados entre 20 e 40 anos. As diferenças relativas são similares entre os sexos até a idade 
GOTLIEB, S. L. D. Mortalidade diferencial por causas, São Paulo, Brasil, 1970: tábuas de vida de múltiplo decremento. Rev. Saúde públ., S Paulo, 15:401-17, 1981.

de 75 anos e a partir daí é no sexo feminino que se acentua o papel predominante das causas cerebrovasculares na mortalidade tardia, pois haveria um acréscimo de $25 \%$ à esperança de vida.

É indiscutivel que o cálculo das tábuas de vida, com exclusão do grupo de doenças cardiovasculares como causa de morte, é, até certo ponto, um exercício teórico e hipotético, pois a eliminação completa é praticamente impossivel, até o presente momento. Entretanto, os ganhos de 9,14 e 11,44 anos têm caráter didático, no sentido de que mostram a importância desse grupo de doenças como causa de morte. Não há dúvidas que, em sociedades de maior poder aquisitivo, a doença isquêmica do coração é a causa mais importante, tanto de morte como de incapacidade física prematura entre os homens, acarretando uma diminuição de sua contribuição para a sociedade, no momento em que ela é mais valiosa. Este fato é confirmado pelas estatísticas dos países tecnologicamente mais avançados que, em 1979 , tiveram $39 \%$ dos óbitos masculinos entre 25 e 64 anos causados por doenças cardiovasculares.

Embora se reconheça a impossibilidade destas causas serem totalmente evitadas, é importante pensar em postergá-las, fazendo com que não ocorram em idades jovens. Estudos epidemiológicos permitem detectar alguns fatores de risco dessas doenças, principalmente das isquêmicas do coração e cerebrovasculares. Se houvesse prevenção atuante sobre estes fatores (hábito de fumar, obesidade, consumo de determinados tipos de gorduras, vida sedentária e estresse ${ }^{39}$ ), poder-se-ia proteger $o$ individuo suscetível. Não resta a menor dúvida que esta atuação ainda é a nivel individual dada a grande dificuldade de atuação a nível populacional 19 .

Outro nível de prevenção, o secundário, de acordo com Leavell e Clarck ${ }^{23}$, seria o diagnóstico e tratamento precoces. Esta atuação ainda seria irreal a nivel populacional dada a atual situação de saúde do município de São Paulo, onde existem outras prioridades. A nivel individual, entretanto, através da atuação de assistência médica eficiente e eficaz é possivel acreditar na sua existência, postergando o momento da morte.

\subsubsection{Eliminando o Grupo dos Acidentes, Envenenamentos e Violências}

A mortalidade por esse grupo de causas externas merece atenção por contribuir, cada vez mais, para a mortalidade em todas as idades, destacando-se a faixa etária de maior produtividade - a população economicamente ativa ${ }^{37}$.

Em 1970, no município de São Paulo, este grupo de causas foi responsável por $12,55 \%$ dos óbitos masculinos (risco de 11,19 por dez mil homens) e $4,62 \%$ dos óbitos femininos (risco de morte de 2,98 por dez mil mulheres).

Ao se comparar as probabilidades de morte real e hipotética - na ausência de causas violentas - segundo sexo e idade. confirma-se o grande impacto desse grupo na mortalidade masculina. A probabilidade de morrer na idade de um ano estaria reduzida em $5 \%$ e na faixa de 15 a 24 anos haveria um decréscimo de $65 \%$. No sexo feminino, apesar das diferenças absolutas serem menores, dado o menor risco de morte entre mulheres, as diferenças relativas têm valores apreciáveis entre 4 e 44 anos (no grupo de 15 a 19 anos a redução é de $40 \%$ ).

A probabilidade de sobreviver dos 15 aos 65 anos passaria de $61,87 \%$ para $66,87 \%$ nos homens e de $77,29 \%$ para $77,63 \%$ nas mulheres.

As tábuas de múltiplo decremento chegam às estimativas das esperanças de vida mos- 
GOTLIEB, S. L. D. Mortalidade diferencial por causas, São Paulo, Brasil, 1970: tábuas de vida de múltiplo decremento. Rev. Saúde públ., S Paulo, 15:401-17, 1981.

trando ganhos de 2,36 anos para os homens e 0,77 anos para as mulheres.

Dado o fato desse grupo de causas apresentar uma complexidade de situações, analisou-se cada uma delas, "de per si".

Os acidentes de veículos a motor compreendem a maior proporção das mortes por causas violentas $(42 \%)$ e vem aumentando significativamente no decorrer dos anos (em 1970 o risco de morrer foi o dobro do valor de 1960). A par da mortalidade existe uma alta morbidade, pois enquanto em 1970 no município de São Paulo houve 1.740 óbitos por acidentes de veículos a motor, o número de vítimas foi igual a 21.235 , representando um risco 358 vitimas para cem mil habitantes 21 .

As probabilidades de morte hipotéticas estariam reduzidas em $9,71 \%$ para meninos de 3 anos, chegando ao máximo de $22,77 \%$ na faixa de 5 a 9 anos.

Os valores relativos são altos até 49 anos, quando outras causas de morte passam a desempenhar papel mais proeminente. No sexo feminino as diferenças absolutas são menores e é no grupo de 15 a 19 anos que haveria a maior diminuição $(20 \%)$.

A esperança de vida ao nascer mostra ganhos de 0,95 anos e 0,35 anos respectivamente para os homens e mulheres. A possivel solução desse problema de Saúde Pública é bastante complexa dada a existência de uma multiplicidade de circunstâncias e fatores ambientais em relação à causalidade desses acidentes. Há necessidade de esforços em várias áreas de atuação: transportes, engenharia, educação, atenção médica, quer preventiva quer curativa, e policiamento.

A categoria "outros acidentes" representou $27,86 \%$ e $26,57 \%$ dos óbitos violentos masculinos e femininos; na idade de um ano esta proporção atingiu os valores de $70 \%$ e $92,31 \%$ respectivamente. Estes óbitos refletem, principalmente, os acidentes domésticos, isto é, envenenamentos acidentais, acidentes por fogo, afogamentos e quedas. Desta maneira, a redução de $5 \%$ e $4 \%$ na probabilidade de morte dessas crianças seria altamente viável desde que existissem programas educativos voltados à prevenção de acidentes domésticos, reconhecidos como verdadeira epidemia dos tempos modernos ${ }^{5}$. Nos adolescentes, o problema muda de aspecto: representaram estes acidentes $47,2 \%$ dos óbitos violentos; deixam de ser domésticos e passam a ser acidentes do lazer (afogamentos) e acidentes do trabalho. Esta é a faixa etária em que os jovens se introduzem na força do trabalho ${ }^{37} \mathrm{e}$, dado talvez sua inexperiência, estariam mais expostos aos riscos de acidentar-se gravemente no ambiente de trabalho. Recomenda Berlinck ${ }^{1}$ a necessidade da realização, com mais intensidade e constância, de cursos específicos de segurança do trabalho.

Os suicídios e lesões auto-infligidas são importantes como causas de morte entre as mulheres, a partir de 15 anos, representando $20 \%$ do total de óbitos violentos.

Os homicidios se tornam importante causa de morte para os homens na idade de 15 a 19 anos ( $21 \%$ dos óbitos violentos). Esta idade representa o marco inicial deste problema de caráter social pois, nas idades sucessivas, avoluma-se a percentagem de mortes por homicídio.

\subsubsection{Análise Global}

Após a análise isolada da influência de cada um dos grupos selecionados de doenças como fator de risco de morte dos residentes no município de São Paulo, em 1970, há necessidade de uma visão conjunta destes aspectos. Nas Tabelas 2 e 3 são apresentadas as diferenças relativas $(\%)$ entre as 
GOTLIEB, S. L. D. Mortalidade diferencial por causas, São Paulo, Brasil, 1970: tábuas de vida de múltiplo decremento. Rev. Saúde públ., S. Paulo, 15:401-17, 1981.

probabilidades real e hipotética de morte, segundo faixa etária. Em cada faixa etária foi possivel apontar o principal grupo de causas e, conseqüentemente, blocos se delinearam. A mortalidade diferencial entre sexos e nas idades pode ser confirmada e um bom exemplo é a atuação das neoplasias malignas, pois constituiram-se na segunda causa de óbito para mulheres com mais de 35 anos e com uma intensidade de atuação muito próxima a das cardiovasculares, fato não verificado entre os homens.

T A B E L A 2

Diferenças relativas $(\%)$ entre as probabilidades real e liquida de morte no sexo masculino, segundo faixas etárias e grupos de causas de morte eliminadas.

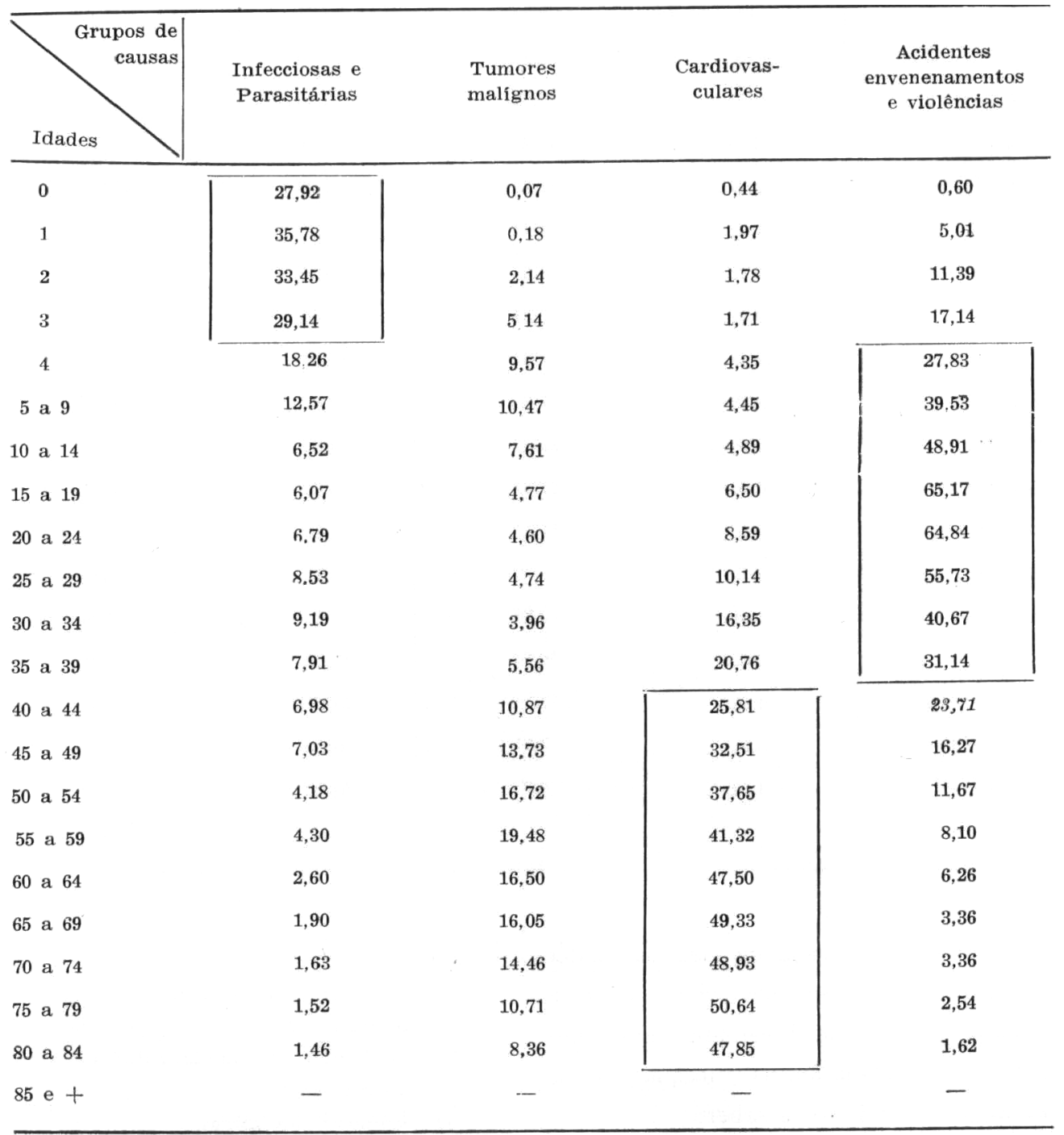


GOTLIEB, S. L. D. Mortalidade diferencial por causas, São Paulo, Brasil, 1970: tábuas de vida de múltiplo decremento. Rev. Saride públ., S Paulo, 15:401-17, 1981.

T A B E L A 3

Diferenças relativas $(\%)$ entre as probabilidades real e líquida de morte no sexo feminino, segundo faixas etárias e grupos de causas de morte eliminadas.

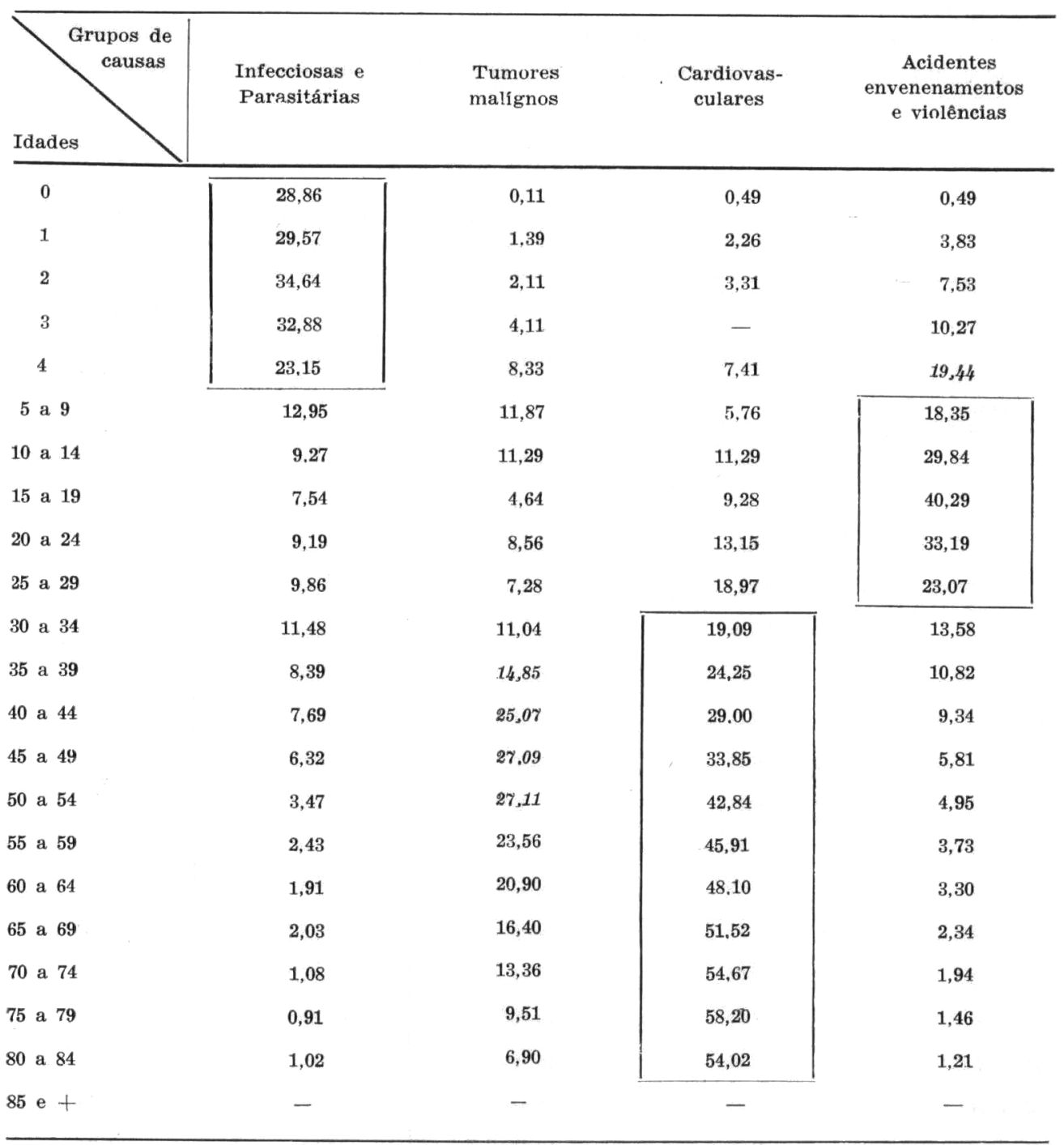

A variável esperança de vida ao nascer sintetiza e expressa nun único valor a atuação das doenças e permitiu apresentar, por ordem de atuação, as principais causas de morte (Tabela 4), a saber: a) no sexo masculino:

1) doenças cardiovasculares,

2) doenças infecciosas e parasitárias,

3) acidentes, envenenamentos $e$ violências $e$

4) tumores malignos; 
GOTLIEB, S. L. D. Mortalidade diferencial por causas, são Paulo, Brasil, 1970: tábuas de vida de múltiplo decremento. Rev. Saúde públ., S. Paulo, 15:401-17, 1981.

b) no sexo feminino:

1) doenças cardiovasculares,

2) doenças infecciosas e parasitárias,
3) tumores malígnos e

4) acidentes, envenenamentos $e$ violências.

T A B E I A 1

Número de anos ganhos na esperança de vida ao nascer, segundo sexo e grupos de causas eliminados.

\begin{tabular}{|c|c|c|}
\hline $\begin{array}{l}\text { Grupos de } \\
\text { causas eliminados }\end{array}$ & Masculino & Feminino \\
\hline Infecciosas e Parasitárias & $2.52(2)$ & $2,24(2)$ \\
\hline Cáncer & $1.87(4)$ & $2,02(3)$ \\
\hline Cardiovasculares & $0.14(1)$ & $11,44(1)$ \\
\hline $\begin{array}{l}\text { Acidentes, Envenenamentos } \\
\text { e Violências }\end{array}$ & $2,36(3)$ & $0,77(4)$ \\
\hline
\end{tabular}

( ) Representa a ordenação do grupo de causas.

\subsubsection{Consideraçōes Finais}

A análise desses resultados conduz à constatação de que houve dualismo na natureza dos principais grupos de causas de morte dos residentes no municipio de São Paulo em 1970. Se por um lado doenças crônico-degenerativas propiciariam grandes ganhos à esperanças de vida hipotéticas, por outro lado as doenças infecciosas (transmissiveis e evitáveis) também assim atuariam caso não existissem como causa de morte. Esses resultados quando comparados a ganhos de outros paises (Tabelas 5 e 6) levam a qualificar o municipio de São Paulo ora como uma região de acordo com o padrão de nível de saúde de países desenvolvidos, ora como uma região em desenvolvimento.

T A B E L A 6

\begin{tabular}{lcccc}
$\begin{array}{l}\text { Esperança de vida ao nascer no sexo masculino e ganhos (em anos) após } \\
\text { eliminaça de alguns }\end{array}$ & $\begin{array}{c}\text { grupos de causas de morte, segundo paises. em 1968, e } \\
\text { municiplo de São }\end{array}$ & $\begin{array}{c}\text { Paulo, em } \\
1970 .\end{array}$ \\
\hline Pais/Municipio & $\begin{array}{c}\text { Esperança de } \\
\text { vida ao nascer }\end{array}$ & $\begin{array}{c}\text { Ganho quando } \\
\text { eliminado o grupo } \\
\text { das cardiovasculares }\end{array}$ & $\begin{array}{c}\text { Ganho quando } \\
\text { eliminado o grupo } \\
\text { das neoplasias } \\
\text { malignas }\end{array}$ \\
\hline Canadá & 69,1 & 10,1 & 2,4 \\
Estados Unidos & 66,6 & 12,1 & 2,3 \\
Israel & 70,5 & 10,4 & 2,2 \\
Colômbia & 59,0 & 4,3 & 1,3 \\
México & 60,5 & 2,1 & 0,8 \\
Mun. S. Paulo & 60,1 & 9,1 & 1,9 \\
\hline
\end{tabular}

Fonte: World Health Statistics Report 28. 
GOTLIab, S. L. D. Mortalidade diferencial por causas, São Paulo, Brasil, 1970: tábuas de vida de múltiplo decremento. Rev. Saúde públ., S Paulo, 15:401-17, 1981.

T A B E L A 6

\begin{abstract}
Esperança de vida ao nascer no sexo feminino e ganhos (em anos) após eliminação de alguns grupos de causas de morte, segundo países. em 1968, e município de São Paulo, em 1970.
\end{abstract}

\begin{tabular}{lccc}
\hline País/Municiplo & $\begin{array}{c}\text { Esperança de } \\
\text { vida ao nascer }\end{array}$ & $\begin{array}{c}\text { Ganho quando } \\
\text { eliminado o grupo } \\
\text { das cardiovasculares }\end{array}$ & $\begin{array}{c}\text { Ganho quando } \\
\text { eliminado o grupo } \\
\text { das neoplasias } \\
\text { malignas }\end{array}$ \\
\hline Canadá & 75.7 & 12,2 & 2,8 \\
Estados IJnidos & 74,1 & 15,3 & $\mathbf{2 , 5}$ \\
Israel & 73,7 & 13,1 & $\mathbf{2 , 5}$ \\
Colómbia & 62,4 & 4,6 & 1,7 \\
México & 63,8 & 2,1 & 1,2 \\
Mun. S. Paulo & 67,2 & 11,4 & 2,0 \\
\hline
\end{tabular}

Fonte: World Health Statistics Report $2 x$.

Os valores das esperanças de vida da população em estudo se aproximam mais de valores apresentados pela Colômbia e México, distanciando-se dos valores apresentados pelo Canadá, EUA e Israel. Por outro lado, os ganhos nas esperanças de vida, com eliminação das doenças cardiovasculares e do câncer, estão mais próximos dos ganhos do Canadá, EUA e Israel, do que aos do México e Colômbia.

A mortalidade proporcional por doenças infecciosas e parasitárias " no Canadá, EUA e Israel foi, respectivamente, igual a $0,8 \%$, $0,9 \%$ e $1,1 \%$, e na Colômbia e México as mesmas foram iguais a $10,7 \%$ e $9,7 \%$. Portanto, o municipio de São Paulo, com $11,6 \%$, encontrava-se em 1970 , com problemas de saúde semelhantes aos países em desenvolvimento, onde as doenças infecciosas e parasitárias, apesar de plenamente evitáveis, ainda propiciavam altos coeficientes de mortalidade nas idades muito jovens.

Por sua vez, os problemas de saúde de paises altamente desenvolvidos também se apresentaram no município de São Paulo.
Atuaram, provavelmente, na parcela da população de nível sócio-econômico mais alto que conseguindo sobreviver às idades mais jovens, veio se expor aos possíveis fatores de risco das doenças degenerativas e crônicas. Esses problemas de saúde ainda estariam agravados por possiveis falhas existentes no sistema de atenção médica do município de Sáo Paulo que ocasionariam mènor sobrevivência aos doentes diagnosticados, refletindo menor ganho nas esperanças de vida ao nascer (Tabelas 5 e 6).

Face a essas considerações, pode levantar-se a hipótese de que o padrão de mortalidade no município de São Paulo, em 1970, indicaria a existência de problemas de saúde de uma população formada por distintos estratos e não de uma população homogênea; tais estratos apresentariam problemas específicos e inerentes a cada um desses segmentos da população. Não se pode esquecer que os coeficientes gerais resultam de uma média ponderada entre os valores apresentados pelos setores sócio-econômicos mais favorecidos e por aqueles com condições muito mais adversas.

* Os dados de morte segundo Idade e causa foram retirados do Demographic Yearbook 6, 1969 e a partir destes os indices foram calculados. 
GOTLIEB, S. L. D. Mortalidade diferencial por causas, Săo Paulo, Brasil, 1970: tábuas de vida de múltiplo decremento. Rev. saúde pübl., S. Paulo, 15:401-17, 1981.

Portanto, há de ser lembrada esta limitação ao serem usados, como subsídios, em diagnóstico de nível de saúde de uma área, para a decisão de ações prioritárias.

Recomenda-se então, que sejam feitos estudos de mortalidade, procurando sempre estabelecer, distintamente, a situação sócio-econômica e sanitária desses setores da população. Por si mesmas, tais observações em nada melhorariam a real situaçāo dos grupos menos favorecidos, porém poderiam evidenciar as desigualdades que existem dentro de uma região. A finalidade em assinalá-las é, pelo menos, como Somoza ${ }^{38}$ recomenda ". . poder conscientizar as pessoas menos informadas e provocar interesse para que sejam adotadas medidas para melhorar a situaçăo.

\section{CONCLUSöES}

1. A esperança de vida ao nascer para os residentes no município de São Paulo, em 1970 , foi igual a 63,55 anos $(60,12$ anos no sexo masculino e 67,21 anos no sexo feminino).
2. A ordenação dos principais grupos de doenças e agravos à saúde, em função dos ganhos propiciados à esperança de vida ao nascer, caso não tivessem sido fator de risco de morte, foi a seguinte:

- Sexo Masculino: doenças cardiovasculares $(9,14$ anos $)$; doenças infecciosas e parasitárias (2,52 anos); acidentes, envenenamentos e violências (2,36 anos); tumores malígnos ( 1,87 anos);

- Sexo Feminino: doenças cardiovasculares (11,44 anos); doenças infecciosas e parasitárias $(2,24$ anos); tumores malígnos (2,02 anos); acidentes, envenenamentos e violências $(0,77$ anos).

3. O padrão de mortalidade no municipio de São Paulo, em 1970, indicaria a existência de problemas de saúde de uma população formada por setores distintos onde coexistiriam condições adversas à saúde, típicas ora de regiñes consideradas desenvolvidas, ora de regiōes em desenvolvimento.

GOTLIEB, S.L.D. [Mortality differentials classified according to cause of death, S. Paulo, Brazil, 1970. Multiple decrement life tables]. Rev. Saúde públ., S. Paulo, $15: 401-17,1981$.

ABSTRACT: General considerations about multiple decrement life tables are made to evaluate the impact of some diseases and other causes of death on the probabilities of death and survival and on the life expectancy of the inhabitants of the City of S. Paulo, Brazil, in 1970. The male life expectancy calculated was 60.12 years, and the female, 67.21. The main groups of causes of death, classified by the gains, were: for males-cardiovascular diseases, infectious and parasitic diseases, accidents, poisonings and violences and neoplasms; for women-cardiovascular diseases, infectious and parasitic diseases, neoplasms and accidents, poisonings, and violence. A dichotomous population could be considered as an explanatory hypothesis for the pattern of mortality prevailling in 1970 in $\mathrm{S}$. Paulo; that is, the patterns probably reflect the health problems of under developed countries as well as those of developed countries.

UNITERMS: Mortality, S. Paulo, SP, Brazil. Survival. Life expectancy. 
GOTLIEB, S. L. D. Mortalidade diferencial por causas, Săo Paulo, Brasil, 1970: tábuas de vida de múltiplo decremento. Rev. Saúde públ., S. Paulo, 15:401-17, 1981.

\section{REFERENCIAS BIBLIOGRAFICAS}

1. BERLINCK, E.L. Acidentes do trabalho; Conferéncla proferida no C:rso de Inspetores de Segurança do Trabalho promovido pelo Instituto Brasileiro de Segrrança de São Paulo. O Estado de São Paulo, S. Paulo, 03 maio 1971.

2. BRESLOW, L. Early case - finding, treatment and mortality from cervix and breast cancer. Prev. Med., 1:141-52, 1972.

3. CERISOLA, M.J.E. Republica Argentina: analisis de la mortalidad por causa (especial referencia al periodo 1960-1966). In: Conferencia Regional Latinoamericana de Población, México, 1970. Actas 1. México, El Colegio de México, 1972. p. 127-32.

4. CHIANG, C.L. Introduction to stochastic processes in biostatistics. New York, John Wiley, 1968.

5. COBLENTZ,A. Les accidents survenumt aux enfants dans le logement ou dans son proche environnement: causes, consequences, prevention. Paris, Anthropologie Appliquée, 1974 (Doc. A.A. 66/74).

6. DEMOGRAPHIC YEARBOOK: 1969. (United Nations) New York, 1970.

7. DOLL, R. Epidemiology of cancer: current perspectives. Amer. J. Epidem., 104:396$-404,1976$.

8. DORN, H. Mortality. In: Hauser, P.M. \& Duncan. O.D. The study of population: an inventory and appraisal. Chicago, University of Chicago Press, 1959. p. 437-71.

9. DUBLIN, L.I. \& LOTKA, A.J. Uses of life table in vital statistics. Amer. $J$. publ. Flth, 27:481-91, 1987.

10. DUBLIN, L.I. et al. Lenght of life: a study of the life table. New York. The Ronald Press, 1949..

11. FRaUmenI Jr., J.F. Persons at high risk of cancer: an approach to cancer etiology and control. New York, Academic Press. 1975.

12. FUNDAÇAO IBGE. Censo demografico: Säo Paulo. Rio de Janeiro, 1973. Tomo 18, v. 1. (VIII Recenseamento Geral do Brasil, 1970).
13. FUNDAÇAO IBGE. Estado de São Paulo: populacão presente na data do censo, 1960. Rio de Janeiro, s.d. [mimeografado]

14. GABALDON, A. Changing problems of preventive medicine in the tropics. In: Conference of the Industrial Council for Tropical Health, 4th, Boston, 1960. Boston, Harvard School of Public Health, 1961. p. 14-28.

15. HEALTH trends and prospects: $1950-$ 2000. Whd Hith Statist. Rep., Geneva, 27:670-706, 1974.

16. HIGGINSON, J. Cancer etiology and prevention. In: Fraumeni Jr., J.F. Persons at high risk of cancer: an approach to cancer etiology and control. New York, Academic Press, 1975. p. 385-98.

17. KARN, M.N. An inquiry into variots death-rates and the comparative influence of certain diseases in the duration of life. Ann. Eugen., London, 4:279-326, 1931.

18. KIMBALL, A.W. Models for the estimation of competing risks from grouped data. Biometrics, $25: 329-37,1969$.

19. LAURENTI, R. Coronariopatias: aspectos epidemiologicos, fatores de risco e prevencão. São Paulo, Faculdade de Saúde Pública USP, 1975. [mimeografado]

20. LAURENTI, R. Fatores de erro na mensuração da mortalidade infantil. Rev. Saúde públ., S. Paulo, 9:529-37, 1975.

21. LAURENTI, R. et al. Alguns aspectos epidemiológicos da mortalidade por acidentes de trânsito de veículos a motor na cldade de São Paulo. Rev. Saúde públ., S. Paulo, 6:329-41, 1972.

22. LAUURENTI, $R$. et al. Alguns aspectos particulares referentes aos resultados da Investigaça Interamericana de Mortalidade na Infancia na área do projeto de São Pa:ılo, Brasil. Bol. Ofic. sanit. panamer., 79:1-14, 1975.

23. LEAVELL, H. \& CLARK, E. Medicina preventiva. São Paulo, McGraw Hill do Brasil, 1976. p. 17.

24. LESER, W.S. A mortalidade infantil no periodo de 1950 a 1970: influência dos fatores demográficos, sócio-econômicos e ambientais no nível de saúde. Probl. bras., 12(134):17-29, 1974. 
GOTLIEB, S. L. D. Mortalidade diferencial por causas, São Paulo, Brasil, 1970: tábuas de vida de múltiplo decremento. Rev. Saúde pübl., S. Paulo, 15:401-17, 1981.

25. LESER, W.S. \& BARBOSA, V. Relacionamento de certas características populacionais com a mortall lade infantil no municlpio de São Paulo de 1950 a 1970. Probl. bras., 10(109):17-30, 1972.

26. LEVIN, M.L. Discussion of "Epidemiology of cancer: current perspectives". Amer. J. Epidem., $104: 405-7,1976$.

27. LEVIN, L.D. et al. Cancer rates and risks. Washington, D.C., Public Health Service. National Instit: te of Health, 1974. (DHEW Publ. (NIH) 75-691).

28. LIFE tables. Wld Hlth Statist. Rep., Geneva, 25:430-42, 1972.

29. ORGANIZAÇÃO PANAMERICANA DA SAUDE. Manual de classificagão esta. tistica internacional de doenças, lesōes $e$ causas de b́bito: $8^{\circ}$ Revisão, 1965. Washington, D.C., 1969. (Publ. cient., 190).

30. OYA, D.R.T. Estudo da distribuigão do fator de separasāo $\mathrm{f}_{\mathrm{x}}^{\mathrm{t}}$ na tábua de sobrevivencia. São Paulo, 1970. [Dissertação de Mestrado - Facul lade de Saúde Pública da USP]

31. PRESSAT, R. Mortality projection and actual trends: a comparative study. Wld Hlth Statist. Rep., Geneva, 27:516-39, 1974.

32. PUFFER, R.R. \& SERRANO, C.V. Caracteristicas de la mortalidad en la niñez. Washington, D.C., Organización Panamericana de la Salud, 1973. (OPAS - Publ. cient., 262).
33. RAMOS, R. Indicadores do nivel de saúde: sua aplicąão ao municipio de São Paulo (1894-1959). São Paulo, 1962. [Tese de Doutoramento - Faculdade de Saúde Pública da USP]

34. SCHNEIDERMAN, M. Sources, riscurces and tso ris. In: Fraumeni Jr., J.F. Persons at high risk of cancer: an approach to cancer etiology and control. New York, Academic Press, 1975. p. 451-66.

35. SECRETARIA DE ECONOMIA E PLANEJAMENTO. Recursos humanos da Grande São Paulo. São Paulo, 1971.

36. SHRYOCK, J.S. et al. The methods and materials of demography. Washington, D.C., U.S. Government Printing Office, 1973. v. 2.

37. SILVEIRA, M.H. \& GOTLIEB, S.L.D. Acidentes, envenenamentos e violências como causa de morte dos residentes no municıpio de São Paulo, Brasi.. Rev. Saúde pübl., S. Pauı, 10:45.55, 1976.

38. SomozA, J.L. La mortali ad en America Latina. In: Conferencia Regional Latino-americana de Población, México, 1970. Actas 1. México, El Colegio de México, 1972. p. 3-12.

39. STAMLER, J. Cardiologia preventiva. Bar celona, Ed. Científico-Médica, 1970.

40. STATISTICAL BULLETIN. (Statistical Bureau of the Metropolitan Life Irsurance Co.) New York, 56, Sept. 1975.

41. TODHUNTER, I. A history of the maine matical theory of probabi.ity. New York, Chelsea, 1049.

Recebido para publicasāo em 13/10/1980 Aprovado para publicação em 01/06/1981 